\title{
Numerical analysis of sand/solids production in boreholes considering fluid-mechanical coupling in a Cosserat continuum
}

\author{
André Luís Muller ${ }^{\mathrm{a}}$, Eurípedes do Amaral Vargas Jr., ${ }^{\mathrm{a},}$, Luiz Eloy Vaz ${ }^{\mathrm{b}}$, Rodrigo Pelucci Figueiredo ${ }^{\mathrm{d}}$, \\ Clemente José Gonçalves ${ }^{\mathrm{c}}$ \\ a Pontifical Catholic University of Rio de Janeiro (PUC Rio), Rua Marques de São Vicente 225/301L, 22 453-900, Rio de Janeiro, RJ, Brazil \\ ${ }^{\mathrm{b}}$ Fluminense Federal University (UFF), Niterói, Rio de Janeiro, RJ, Brazil \\ ' Cenpes-Petrobrás, Av. Jequetiba 950, 21941-598, Rio de Janeiro, RJ, Brazil \\ ${ }^{\mathrm{d}}$ Department Mining Engineering, Federal University of Ouro Preto (UFOP), Ouro Preto, Brazil
}

\section{A R T I C L E I N F O}

\section{Article history:}

Received 16 August 2010

Received in revised form

21 July 2011

Accepted 19 September 2011

Available online 13 October 2011

Keywords:

Sand/solids production

Cosserat continua

boreholes

\begin{abstract}
A B S T R A C T
Sand/solids production is a serious problem faced by the petroleum industry during production stages. Particles/groups of particles of the formation rock are produced together with pumped oil or gas causing highly damaging effects on pipes and valves. This phenomena can be caused by several factors including the stresses developed around the boreholes/perforations and fluid flow, which break and erode the rock, respectively. Numerical simulation of sand/solids production presents a considerable challenge as intricacies of failure processes must be correctly simulated. This paper presents a finite element based procedure for simulating the process of sand/solids production, considering fluidmechanical coupling in an elasto-plastic Cosserat continuum. It is believed that the enhanced deformation modes included into Cosserat continua may contribute for proper modeling of the deformational behavior and failure modes of the cemented/non-cemented granular materials involved. Bogdanova-Bontcheva and Lippmann's constitutive model was implemented in order to model the granular materials involved. Finally, the work presents the analysis of sand/solids production in a hypothetical borehole. For comparison purposes, different characteristic lengths of the material are adopted and analysis is also performed using standard continua.
\end{abstract}

(c) 2011 Elsevier Ltd. All rights reserved.

\section{Introduction}

The phenomenon of particle production during the extraction of oil producing wells is commonly called sand or solids production. According to [1], this physical phenomenon usually occurs when the fluid/porous medium, previously stable, becomes unstable, reaches the strength limit of the porous matrix, with consequent breakdown of its constituent parts thereafter. Field observations indicate that perturbations of flow gradients and of effective stress acting on the porous matrix of the formation, initiate tearing of small fractions of the rock.

This phenomenon is commonly seen in sandstones, especially in poorly or non-consolidated ones. However, it does not occur exclusively in these rocks, as it is also observed in rocks of various natures, such as coal and limestones.

Sand production is one of the most frequent and serious problems observed during the extraction of oil or gas. The Society of Petroleum Engineers (SPE) indicates that much world's hydrocarbon reserves are contained in sandstone, and thus potentially

\footnotetext{
* Corresponding author. Tel.: +55 213527 1188; fax: +55 2135271195 .

E-mail address: vargas@puc-rio.br (E.d.A. Vargas Jr.).
}

subject to this phenomenon. Also, if not properly controlled, this might make the development of borehole economically unfeasible, or provide their premature closure. According to [2], it is estimated that seventy percent of the world's hydrocarbon reserves are contained in reservoirs where sand/solids production may occur.

Bianco [3] suggested that the sand/solids production phenomenon in oil producing wells would be associated to three basic sets of factors: magnitude of the in-situ stresses and its variations, pressure gradients, fluid flow velocity and changes in fluid saturation; strength factor (strength of the material, inter-particle friction; arcs of sand, capillary forces); operational factors (strategies of drilling and completion, production procedures and depletion of the reservoir). A description of operational aspects and other mechanisms related to sand/solids production are described in detail in [2].

Some researchers have studied techniques to control the sand/ solids production problem. Additionally, other studies have presented analytical, numerical or laboratory procedures in order to understand and attempts to quantify sand/solids production rates. Amongst the developers of analytical procedures, the works of [4-9] may be cited. On the experimental side, Bianco [3] developed extensive laboratory work to examine the behavior of sand/solids production. On the numerical modeling side, the work of [10-15] may be highlighted and relevant works joining sand/solids production experiments, 
theoretical model development and numerical simulations [16-18]. Despite this already considerable amount of work, correct predictions of sand/solids production rates remain somewhat elusive. Several reasons can be indicated to explain this fact. From the flow/erosion point of view, although many propositions have been presented to quantify sand/solids production rates, not enough experimental or field evidence of their suitability/validity have been put forth. From the mechanical point of view, continua based procedures may not be able to adequately represent deformational behavior occurring in the vicinity of borehole walls especially near failure. An alternative for that is the use of discrete element based methods [19-21] but these methods remain useful for basically for phenomenological modeling, hardly useful for the modeling of real cases. On the other hand, standard continua based methods fail in representing correctly phenomena happening at the microstructural level. A possible alternative to this scenario is the use of enhanced continua such as Cosserat type continua. Cosserat continua were firstly proposed by the Cosserat brothers [22]. The consistent derivation of basic equations of Cosserat elasticity was presented by Mindlin [23]. One of the most important features of the Cosserat continua theory which makes it different from the standard continua is the insertion of couple stresses, which makes the stress tensor non-symmetric [23]. As consequence, in addition to body forces, the Cosserat theory is able to include mass and surface moments, named respectively body couple forces and surface couple forces, which are ignored in the standard theory. At the particle level, an additional degree of freedom, a micro-rotation is introduced. As a consequence, the strain tensor also becomes non-symmetric. This new degree of freedom is micro-polar and produces, as consequence, free spins, i.e., spins which are independent of the media environment. Furthermore, when constitutive equations for Cosserat materials are derived, new parameters, amongst them the characteristic length, appear. This parameter has an important role in the Cosserat theory. Some researchers, [24-27] have established the possibility that the stress tensor can be non-symmetric in granular media, especially cemented granular materials and also that couple stresses are important to understand material instability such as shear banding. Bardet and Vardoulakis [28] have also pointed out at the eventual asymmetries that can be found in stress tensor in granular materials. Therefore, Cosserat continua are presented as a good alternative for the modeling of granular materials, especially granular materials with a certain level of cohesion, given by a cement, as is the case of sandstones.

The present paper presents a finite element implementation of an elasto-plastic Cosserat continuum in an attempt to explore the above mentioned features for the simulation of sand/solids production mechanisms. The constitutive model chosen to represent the elastic-plastic behavior of the material was the Bogdanova-Bontcheva-Lippmann constitutive model, which was specifically developed to represent the behavior of granular materials. The basis for the development of this work was a finite element program previously developed by the authors $[29,30]$, one which allows analysis of poroelastoplasticity problems. Numerical procedures were therefore implemented in that computer program in order to simulate the erosion processes associated with the sand/solids production. The subsequent items describe the implementations carried out, results obtained with the developed program and a pertinent discussion about them.

\section{Development of governing equations}

In this section the formulation for fluid-mechanical coupling in a Cosserat continuum is presented. The equations are described in a domain $\Omega \subset R^{2}$ with a contour $\Gamma$ for a time interval $t \in[0, T]$.

\subsection{Mechanical equilibrium equations}

The equilibrium equation, Eq. (1), is obtained through the virtual work principle for static problems. This equation relates the real quantities, as the total stress $\boldsymbol{\sigma}$, the body forces $\mathbf{b}$, the surface forces $\mathbf{t}$, the couple stress $\boldsymbol{\mu}$, the body couple forces $\boldsymbol{\Theta}$ and surface couple forces $\tau$ to virtual kinematic quantities as virtual strains $\delta \boldsymbol{\varepsilon}$, virtual displacements $\delta \mathbf{u}$, virtual micro-rotations $\delta \boldsymbol{\omega}^{c}$ and virtual micro-rotations gradients $\delta \mathbf{\kappa}$ :

$\int_{\Omega}\left(\delta \boldsymbol{\varepsilon}^{T} \boldsymbol{\sigma}+\delta \boldsymbol{\kappa}^{T} \boldsymbol{\mu}\right) d \Omega=\int_{\Omega}\left(\delta \mathbf{u}^{T} \mathbf{b}+\delta \boldsymbol{\omega}^{c T} \boldsymbol{\Theta}\right) d \Omega+\int_{\Gamma}\left(\delta \mathbf{u}^{T} \mathbf{t}+\delta \boldsymbol{\omega}^{c T} \tau\right) d \Gamma$

To continue the description of the mechanical equilibrium equation, the following definitions are used: $\overline{\boldsymbol{\sigma}}=\left\{\begin{array}{ll}\boldsymbol{\sigma} & \boldsymbol{\mu}\end{array}\right\}^{T}$, $\overline{\boldsymbol{\varepsilon}}=\left\{\begin{array}{ll}\boldsymbol{\varepsilon} & \boldsymbol{\kappa}\end{array}\right\}^{T}, \overline{\mathbf{b}}=\left\{\begin{array}{ll}\mathbf{b} & \boldsymbol{\Theta}\end{array}\right\}^{T}, \overline{\mathbf{u}}=\left\{\begin{array}{ll}\mathbf{u} & \boldsymbol{\omega}^{c}\end{array}\right\}^{T}$ and $\overline{\mathbf{t}}=\left\{\begin{array}{ll}\mathbf{t} & \boldsymbol{\tau}\end{array}\right\}^{T}$.

Thus, Eq. (1) can be rewritten as

$\int_{\Omega}\left(\delta \overline{\boldsymbol{\varepsilon}}^{T} \overline{\boldsymbol{\sigma}}\right) d \Omega=\int_{\Omega}\left(\delta \overline{\mathbf{u}}^{T} \overline{\mathbf{b}}\right) d \Omega+\int_{\Gamma}\left(\delta \overline{\mathbf{u}}^{T} \overline{\mathbf{t}}\right) d \Gamma$

Total and effective stress and strain are described by [31] as follows:

$\overline{\boldsymbol{\sigma}}=\overline{\boldsymbol{\sigma}}^{\prime}+\mathbf{D} \mathbf{m} \frac{p}{3 K_{s}}-\mathbf{m} p$

$\overline{\boldsymbol{\sigma}}^{\prime}=\mathbf{D} \overline{\boldsymbol{\varepsilon}}+\overline{\boldsymbol{\sigma}}_{0}^{\prime}$

$\bar{\varepsilon}_{i j}=\frac{1}{2}\left(u_{i, j}+u_{j, i}\right)+e_{i j k}\left(\omega_{k}^{c}-\omega_{k}\right)$

In these equations, $\overline{\boldsymbol{\sigma}}^{\prime}$ are the effective stresses, $p$ the pore pressures, $\overline{\boldsymbol{\varepsilon}}$ the skeleton total strain, D the constitutive tensor, $\overline{\boldsymbol{\sigma}}_{0}^{\prime}$ the effective initial stress, $\mathbf{m} p / 3 K_{s}$ the volumetric strain caused by uniform compression of the grains, $K_{s}$ the bulk modulus of solid grains and $\mathbf{m}=\left\{\begin{array}{lllllll}1 & 1 & 1 & 0 & 0 & 0 & 0\end{array}\right\}^{T}$. In Eq. (5), $e_{i j k}$ is the permutation tensor and $\omega_{k}$ is the macro-rotation tensor. For further reading, the work of $[32,33]$ is suggested.

\subsection{Flow equations}

The present development assumes single-phase flow. With the consideration of Darcy's law, the fluid velocity is given by

$\mathbf{v}_{\pi}=-\frac{\mathbf{k}}{\vartheta_{\pi}} \nabla\left(p+\rho_{\pi} g h\right)$

where $\pi$ represents the fluid type, $\rho_{\pi}$ represents the density of the fluid, $\nabla$ the differentiation operator, $\mathbf{k}$ is the absolute permeability tensor of the porous medium, $g$ the gravity acceleration, $h$ is the pressure head and $\vartheta_{\pi}$ the dynamic viscosity of fluid $\pi$.

In a porous medium, fluid flow must satisfy the mass conservation of fluid. The following continuity equation can represent this condition:

$\nabla^{T}\left(\rho_{\pi} q_{\pi}\right)+\dot{m}_{\pi}=0$

where $\dot{m}_{\pi}$ represents the increment of mass of fluid in an infinitesimal portion of the porous medium per time unit and $q_{\pi}$ the fluid discharge.

It is assumed that the porous medium can be described by two parts, one formed by interconnected pores grains occupying a volume $V_{p}$, and another composed of isolated pore and the solid particles, occupying a volume $V_{s}$. The total volume of the porous medium $V$ is given by the sum of the volumes corresponding to each constituent part: $V=V_{p}+V_{s}$.

Assuming also that the porous medium is fully saturated, the volume of fluid $V_{\pi}$, which can circulate freely through the porous medium is equal to the pore volume. The porosity $\phi$ is the ratio 
among pore volume and total volume: $\phi=V_{p} / V$. Therefore, is possible express the mass of fluid per unit volume as $m_{\pi}=\rho_{\pi} \phi$. Thus, $\dot{m}_{\pi}=\dot{\phi} \rho_{\pi}+\dot{\rho}_{\pi} \phi$. The term $\dot{\phi} \rho_{\pi}$ represents the mass change of fluid associated with increased porosity, while the term $\dot{\rho}_{\pi} \phi$ represents the expansion of the fluid.

The variation of the pore volume $V_{p}$ can be decomposed into three parts, the first corresponds to the volumetric variation of the skeleton, the second corresponds to the volume variation of the grains and the third corresponds to the volume generated by sand/solids production. The first two parts are obtained from the fully coupled fluid-mechanical calculations and the third part (the one corresponding to the volume generated to erosion) is calculated in a staggered way.

The volumetric variation of the skeleton is given by

$\frac{d V}{V}=d \overline{\boldsymbol{\varepsilon}}_{v}=\mathbf{m}^{T} d \overline{\boldsymbol{\varepsilon}}$

The variation of the volume of grains is obtained by considering that the grains deform elastically and that the applied load can be decomposed into two parts. The first portion corresponds to the increment of effective stress and the second corresponds to an increase of the confining stress that generates an increase in pore pressure of equal magnitude. Adding the two portions, one obtains the variation of the volume of grains

$\frac{d V_{s}}{V}=\frac{1}{3 K_{s}} \mathbf{m}^{T} \mathbf{D} d \overline{\boldsymbol{\varepsilon}}+\frac{1}{9 K_{s}^{2}} \mathbf{m}^{T} \mathbf{D} \mathbf{m} d p_{\pi}-\frac{(1-\phi)}{K_{s}} d p_{\pi}$

Thus, the rate of the volume, without considering the production of sand/solids, is obtained by $d \phi=d V / V-d V_{s} / V$. In problems with large sand/solids production, this parcel may be insignificant after a long time, but can be significant at other times. After rearranging the terms and time differentiation, the rate of the volume change results in

$\dot{\phi}=\left[\mathbf{m}^{T}-\frac{1}{3 K_{s}} \mathbf{m}^{T} \mathbf{D}\right] \stackrel{\bullet}{\boldsymbol{\varepsilon}}+\left[\frac{(1-\phi)}{K_{s}}-\frac{1}{9 K_{s}^{2}} \mathbf{m}^{T} \mathbf{D} \mathbf{m}+\frac{\phi}{K_{\pi}}\right] \dot{p_{\pi}}$

The fluid density rate is described by $\dot{\rho}_{\pi}=\rho_{\pi} / K_{\pi} \dot{\rho}_{\pi}$, where $K_{\pi}$ is the bulk modulus of the fluid. With the previous equations is defined $\dot{m}_{\pi}$ and finally, considering that the density of the fluid is constant in time $\nabla\left(\rho_{\pi} q_{\pi}\right)=\rho_{\pi} \nabla\left(q_{\pi}\right)$ is written the flow equation:

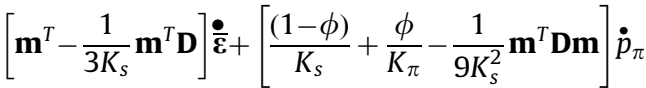

$$
\begin{aligned}
& -\nabla^{T}\left[\frac{\mathbf{k}}{\vartheta_{\pi}} \nabla\left(p_{\pi}+\rho_{\pi} g h\right)\right]=0
\end{aligned}
$$

Additionally, the volume change related to the sand/solids being produced is given by the relationship proposed in [34]:

$\dot{\phi}_{e}= \begin{cases}\lambda(1-\phi) C\left\|\mathbf{v}_{\pi}\right\| & \text { if }\left\|\mathbf{v}_{\pi}\right\| \geq\|\mathbf{v}\|_{\text {critic }} \\ 0 & \text { if }\left\|\mathbf{v}_{\pi}\right\|<\|\mathbf{v}\|_{\text {critic }}\end{cases}$

where $\lambda$ represents the erosion coefficient and $C$ the fluidized sand/solids concentration. The erosion coefficient (having dimensions $L^{-1}$ ), described as a function of plastic strain $\gamma^{p}$, proposed by [17], is described by

$\lambda\left(\gamma^{p}\right)=\lambda_{0}+\frac{\gamma^{p} / \gamma_{\max }^{p}}{\alpha+\beta \gamma^{p} / \gamma_{\max }^{p}}$

where $\gamma^{p}$ is described in terms of a norm of deviatoric plastic strains $\gamma^{p}=\sqrt{2 / 3 e_{i j}^{p} e_{i j}^{p}}$ where $e_{i j}^{p}=\bar{\varepsilon}_{i j}^{p}-1 / 3 \delta_{i j} \bar{\varepsilon}_{v}^{p}$. $\bar{\varepsilon}_{i j}^{p}$ represents the plastic strains, $\delta_{i j}$ the Krönecker delta tensor and $\bar{\varepsilon}_{v}^{p}$ the volumetric plastic strains. As $\overline{\boldsymbol{\varepsilon}}=\left\{\begin{array}{ll}\boldsymbol{\varepsilon} & \boldsymbol{\kappa}\end{array}\right\}^{T}$, in a Cosserat continuum, the erosion coefficient, shown above, is a function of the symmetric and non-symmetric strains and also of the micro-rotations gradients. $\lambda_{0}, \alpha$ and $\beta$ are constants to be experimentally determined as proposed in [17]. The variable $\gamma_{\max }^{p}$ represents the maximum value of the $\gamma^{p}$ in domain, at each time step.

\section{Fluid-mechanical coupling and finite element formulation}

For the formulation of the equations described in the previous paragraph using the finite element method, the traditional interpolation matrices for displacements, micro-rotations and pore pressures, respectively, $\mathbf{N}_{u}$ and $\mathbf{N}_{p}$, are used. These matrices relate the values of the variables at the element nodes to the ones at an interior point of an element. From the former, the straindisplacement matrix $\mathbf{B}$ can be derived.

The constitutive tensor for an elastic Cosserat continuum contains additional terms, $\varsigma^{c}$ and $\eta$ linked to the relationships between couple stresses and rotations [35]. $\varsigma^{c}$ is a Cosserat constant, similar to the shear modulus and $\uparrow$ a characteristic length of the material. The presence of the characteristic length is one of the main differences between the standard and the Cosserat continua. In standard continua, the medium is a continuous distribution of particles, each represented geometrically by a material point. In the Cosserat continua, each point can be seen as a particle of small size, which is, itself, a small continuous around the point. If the characteristic length is negligible, the Cosserat continuum takes the characteristics of a standard continuum.

For the time domain discretization of the variables $\mathbf{u}$ - and $\mathbf{p}$, the generalized trapezoidal rule suggested in [36] is used. Thus the following equations can be obtained:

$$
\begin{aligned}
{ }^{\mathrm{t}+}+\Delta \mathrm{t} & \mathbf{F}_{u}\left({ }^{\mathrm{t}+\Delta \mathrm{t}} \overline{\mathbf{u}}^{\mathrm{t}+\Delta \mathrm{t}} \mathbf{p}, t\right) \\
= & \int_{\Omega} \mathbf{B}^{T} \mathbf{D} \mathbf{B} d \Omega^{\mathrm{t}+\Delta \mathrm{t}} \overline{\mathbf{u}}+\int_{\Omega} \mathbf{B}^{\mathrm{T}} \mathbf{D} \frac{\mathbf{m}}{3 K_{s}} \mathbf{N}_{p} d \Omega^{\mathrm{t}+\Delta \mathrm{t}} \mathbf{p} \\
& -\int_{\Omega} \mathbf{N}_{u}{ }^{T} \overline{\mathbf{b}} d \Omega-\int_{\Gamma} \mathbf{N}_{u}{ }^{T} \overline{\mathbf{t}} d \Gamma-\int_{\Omega} \mathbf{B}^{\mathrm{T}} \mathbf{m} \mathbf{N}_{p} d \Omega^{\mathrm{t}+\Delta \mathrm{t}} \mathbf{p}+\int_{\Omega} \mathbf{B}^{T} \overline{\boldsymbol{\sigma}}_{0}^{\prime} d \Omega
\end{aligned}
$$

$$
\begin{aligned}
{ }^{\mathrm{t}+\Delta \mathrm{t}} \mathbf{F}_{p}\left({ }^{\mathrm{t}+\Delta \mathrm{t}} \overline{\mathbf{u}}^{\mathrm{t}+\Delta \mathrm{t}} \mathbf{p}, t\right) \\
=\int_{\Omega}\left(\nabla \mathbf{N}_{p}\right)^{T} \frac{\mathbf{k}}{\vartheta_{\pi}} \nabla \mathbf{N}_{p} d \Omega\left[(1-\theta)^{t} \mathbf{p}+\theta^{\mathrm{t}+\Delta \mathrm{t}} \mathbf{p}\right] \\
\quad+\int_{\Omega}\left(\nabla \mathbf{N}_{p}\right)^{T} \frac{\mathbf{k}}{\vartheta_{\pi}} \nabla \rho_{\pi} g h d \Omega+\int_{\Omega} \mathbf{N}_{p}{ }^{T} s \mathbf{N}_{p} d \Omega\left(\frac{{ }^{\mathrm{t}+\Delta \mathrm{t}} \mathbf{p}-{ }^{t} \mathbf{p}}{\Delta t}\right) \\
\quad+\int_{\Omega} \mathbf{N}_{u}{ }^{\mathrm{T}}\left(\mathbf{m}^{T}-\frac{\mathbf{m D}}{3 K_{s}}\right) \mathbf{B} d \Omega\left(\frac{\mathrm{t}+\Delta \mathrm{t} \overline{\mathbf{u}}-{ }^{t} \overline{\mathbf{u}}}{\Delta t}\right)+\int_{\Gamma} \mathbf{N}_{p}{ }^{T} q_{\pi} d \Gamma
\end{aligned}
$$

where $\quad s=\left((1-\phi) / K_{s}\right)+\left(\left(\phi / K_{\pi}\right)-1\right) / 9 K_{s}^{2} \mathbf{m}^{T} \mathbf{D m}$, and ${ }^{\mathrm{t}+\Delta \mathrm{t}} \mathbf{F}_{u}$ $\left({ }^{\mathrm{t}+\Delta \mathrm{t}} \overline{\mathbf{u}},{ }^{\mathrm{t}+\Delta \mathrm{t}} \mathbf{p}, t\right)$ and ${ }^{\mathrm{t}+\Delta \mathrm{t}} \mathbf{F}_{p}\left({ }^{\mathrm{t}+\Delta \mathrm{t}} \overline{\mathbf{u}},{ }^{\mathrm{t}+\Delta \mathrm{t}} \mathbf{p}, t\right)$ represent residual vectors ${ }^{t}+\Delta t \mathrm{R}$ at the end of the time interval once the values of $\overline{\mathbf{u}}$ and $\mathrm{p}$ at the beginning of the time interval are known. In order to solve Eqs. (14) and (15), the unknown vectors ${ }^{t+\Delta t} \overline{\mathbf{u}}$ and ${ }^{t+\Delta t}$ p, which make the residual vectors ${ }^{t+\Delta t} \mathbf{R} \cong 0$, must be found. These equations inside an iteration cycle $i$ can be represented in a compact form as.

$$
\begin{aligned}
& {\left[\begin{array}{cc}
-\mathbf{K} & \mathbf{L} \\
\mathbf{L}^{T} & \Delta t \theta \mathbf{H}+\mathbf{G}
\end{array}\right]\left\{\begin{array}{l}
\delta^{\mathrm{t}+\Delta \mathrm{t}} \overline{\mathbf{u}}_{i+1} \\
\delta^{\mathrm{t}+\Delta \mathrm{t}} \mathbf{p}_{i+1}
\end{array}\right\}} \\
& \quad=\left\{-{ }^{\mathrm{t}+\Delta \mathrm{t}} \mathbf{F}_{u}^{i}\left({ }^{\mathrm{t}+\Delta \mathrm{t}} \overline{\mathbf{u}}_{i},{ }^{\mathrm{t}+\Delta \mathrm{t}} \mathbf{p}_{i}, t\right) \Delta t^{\mathrm{t}+\Delta \mathrm{t}} \mathbf{F}_{p}^{i}\left({ }^{\mathrm{t}+\Delta \mathrm{t}} \overline{\mathbf{u}}_{i},{ }^{\mathrm{t}+\Delta \mathrm{t}} \mathbf{p}_{i}, t\right)\right\}
\end{aligned}
$$

In the above equation, the variables are the displacements, micro-rotations and pore pressures increments and the matrices $\mathbf{K}, \mathbf{L}, \mathbf{H}$ and $\mathbf{G}$ are the called tangent matrices.

The porosity is updated, uncoupled of the Eq. (16), at each integration point adding the Eqs. (10) and (12). To compute the sand/solids volume in finite element formulation the Eq. (12) is integrated in each integration point of the element. 
In general, two approaches are employed for the implementation of elasto-plastic constitutive models in Cosserat continua. The first approach ([26,37] among others), is one which deals almost exclusively with bifurcation and localization topics and makes use of a generalization of standard continua plastic flow and plastic potential functions by the introduction, implicitly in the invariants of these functions, the effects generated by couple stresses, anti-symmetric stresses and characteristic lengths. This approach has, typically, being applied in conjunction with von Mises and Mohr Coulomb elasto-plastic models and the generalization is done by modifying the second invariant of deviatoric stresses. This approach has been used as a strategy to resolve computational problems related to the discretization on the numerical solution, by finite elements, of localization problems and not to adequately represent the mechanical behavior of medium. Lippmann [38] and Bogdanova-Bontcheva \& Lippmann [24], on the other hand, introduced an alternative approach, where the particular rotational elastic-plastic behavior of the Cosserat medium is described by introducing a new pair of plastic flow and plastic potential functions. In this approach, standard continua plastic flow and plastic potential functions are modified by adding the effects generated by anti-symmetric stresses. This way, the new and modified plastic flow and potential functions represent the effects of couple stresses, antisymmetric stresses and characteristic length. The studies described in $[38,24]$, focused mainly on the modeling of granular media, better suited therefore for sand/solids production studies. In Bogdanova-Bontcheva \& Lippmann's model, the two sets of functions, plastic flow functions $(f)$ and plastic potential functions (g) are:

$f_{1}=\sqrt{J_{2}}+I_{1} \sin (\theta)+\left(\left|\sigma_{a}\right|-c\right) \cos (\theta)$

$g_{1}=\sqrt{J_{2}}+I_{1} \sin (\psi)+\left(\left|\sigma_{a}\right|-c\right) \cos (\psi)$

$f_{2}=\sqrt{\overline{J_{2}}}-c 7$

$g_{2}=f_{2}$

where $J_{2}$ represents the second invariant of deviatoric symmetrical stress tensor, $\overline{J_{2}}$ represents the second invariant of deviatoric symmetrical couple stress tensor, $I_{1}$ represents the first invariant of symmetrical stress tensor, $\sigma_{a}$ represents the antisymmetric stress, $\sigma_{a}=\left(\sigma_{12}-\sigma_{21}\right) / 2, c$ represents the cohesion of the material, $\theta$ and $\psi$ represent respectively the friction angle and dilatancy angle of the material and $\eta$, as defined above, represents a characteristic length of the material. The plastic flow and plastic potential functions are used as in standard continua for the treatment of surfaces with vertices. When both functions are mobilized, the plastic strain increment is obtained by linear combination of increments for each set of functions. The form given to Eq. (17) has a well established physical motivation, experimentally verified in [24]. The same does not apply to elastoplastic models where Cosserat effects are introduced in the classic invariants [26] whose motivation is purely mathematical. Eq. (17) on the other hand, allow for the consideration, separately, of plastic mechanisms associated to micro-rotations. For sand/solids production phenomena, it has been verified experimentally that these mechanisms may be activated independently [3], that is, not necessarily associated to plastic effects introduced by the classic stress tensors. Therefore, using standard elastoplastic Cosserat models [26] one is not able to consider independently classic plastic mechanisms and Cosserat plastic mechanisms. This is however possible through the use of Eq. (17).

\section{Numerical procedures for the sand/solids production prediction}

It is assumed that the variation of permeability with regard to porosity follows Kozeny-Carman law as follows:

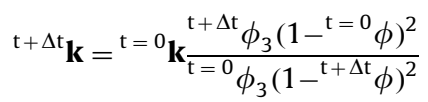

In order to characterize the relationship between mechanical properties and porosity, relations usually used in topological optimization of structures are used. Such relationships are based on a simplification of homogeneization theory [39,40]. Mathematically, the homogeneization theory supports the substitution of composite materials by equivalent materials. In the case of structures optimization, this theory is used for treatment of regions having intermediate densities. In the present work, this theory is adapted for the treatment of regions having intermediate porosities. It is assumed that the microstructure is not known but it is isotropic and that the physical properties are also isotropic and dependent on the porosity. Generally, for the use of homogeneization theory, penalty methods are employed. Amongst these methods, SIMP (Solid Isotropic Microstructure with Penalty) type materials can be used [39].

Using these concepts, cohesion and Young's modulus can be updates in time domain as follows:

${ }^{\mathrm{t}+\Delta \mathrm{t}} V={ }^{\mathrm{t}=0} V\left[1-\left({ }^{\mathrm{t}+\Delta \mathrm{t}} \phi-{ }^{\mathrm{t}=0} \phi\right)\right]^{n}$

where $n$ is a factor that penalizes intermediate porosities. In the present work, $n=2$ is assumed. A summary of the proposed numerical scheme along time can be described as follows. First, obtain displacements, micro-rotations and pore pressures with Eq. (16). Next, update the porosity adding the Eqs. (10) and (12). Then, compute volume of sand/solids being produced with Eq. (12). Update the permeability with Eq. (18) and update cohesion and Young's modulus with Eq. (19). Finally, advance in time and return to step 1 .

\section{Example and discussions}

In order to evaluate the proposed procedure in this work and to verify the consequences of using Cosserat continua in the analysis of sand/solids production, the analysis of a hypothetical vertical borehole is carried out. The geometry of the model is represented by a square of size $6.0 \mathrm{~m} \times 6.0 \mathrm{~m}$, with a circular hole of the $0.20 \mathrm{~m}$ diameter located in the center of the square. The initial conditions in terms of stresses considered equal values in both directions, these correspond to $30.0 \mathrm{MPa}$. An isotropic state of stress was considered as the result of experimental work is available for these conditions and at least qualitative comparisons can be made between numerical and experimental results. The initial pore pressure in rock formation has a magnitude of 22.0 MPa. The mechanical and hydraulic properties necessary for the simulation are presented in Table 1 . The simulation process is carried out in stages beginning with the excavation stage. The excavation stage is considered as instantaneous in time. So, there is no sand/solids production in this state. After this stage, a fluid pressure drawdown of $15 \mathrm{MPa}$ is considered, so a mechanical loading of 7.0 MPa is applied at the borehole wall. The simulation time is five days. For the numerical analysis, a finite element mesh composed by 2800 quadrilateral elements and 2880 nodes was used as shown in Fig. 1.

At this stage it may be necessary to justify the values of the characteristic lengths appearing in Table 1 . It can be shown that the characteristic length in a Cosserat continuum appears in an indirect form. It is given by the square root of the ratio between 
a bending modulus (the one relating couple stresses to curvatures) as shown in [23]. This way, this characteristic length should be related to an assemblage of grains, which would possess a certain bending stiffness as well as introducing a rotational gradient in the medium. In other words, the characteristic length does not represent the dimensions of an individual grain but, necessarily, that of an assemblage of grains. This assemblage

Table 1

Dada for example.

\begin{tabular}{|c|c|}
\hline Parameters & Values \\
\hline${ }^{t=0} E(\mathrm{MPa})$ & $25,000.00$ \\
\hline$v(-)$ & 0.20 \\
\hline${ }^{t}={ }^{0} c(\mathrm{MPa})$ & 5.00 \\
\hline$\theta\left({ }^{0}\right)$ & 30.00 \\
\hline$\psi\left({ }^{0}\right)$ & 30.00 \\
\hline$t=0 \phi(-)$ & 0.20 \\
\hline$t=0 \mathbf{k}\left(\mathrm{m}^{2}\right)$ & $1.00 \mathrm{E}-15$ \\
\hline$\mu$ (MPas) & $1.00 \mathrm{E}-9$ \\
\hline$K_{s}(\mathrm{MPa})$ & $36,000.00$ \\
\hline$K_{\pi}(\mathrm{MPa})$ & 2800.00 \\
\hline$\lambda_{0}\left(m^{-1}\right)$ & 1.80 \\
\hline$\alpha(\mathrm{m})$ & 0.006 \\
\hline$\beta(\mathrm{m})$ & 0.20 \\
\hline$\|\mathbf{v}\|_{\text {critic }}(\mathrm{m} / \mathrm{s})$ & 0.00 \\
\hline$C(-)$ & 0.05 \\
\hline$\varsigma^{c}(\mathrm{MPa})$ & 5000.00 \\
\hline$\eta(\mathrm{m})$ & $0.01 ; 0.02$ \\
\hline
\end{tabular}

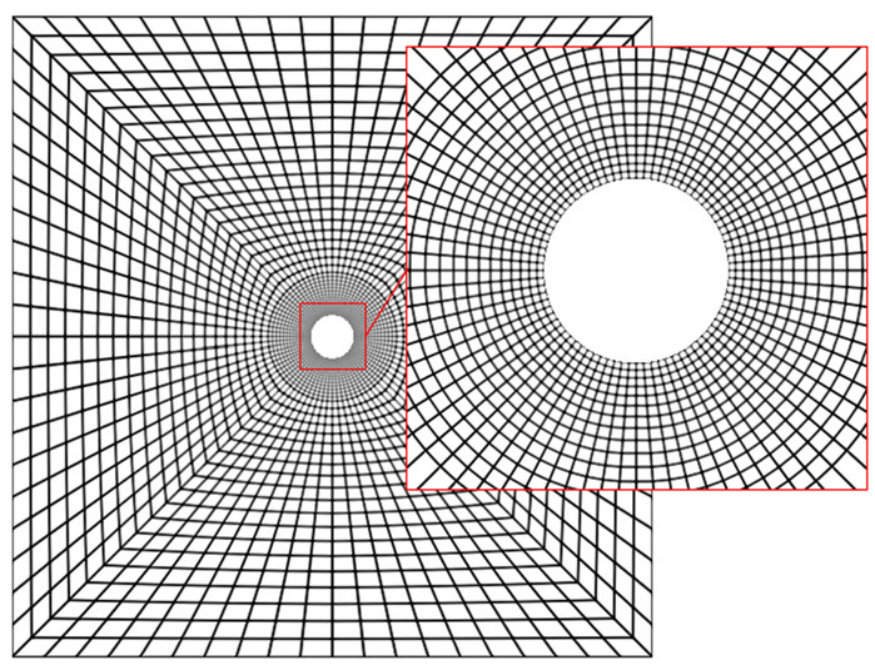

Fig. 1. Finite element mesh of borehole geometry. defines a sort of representative elementary volume for the continuum one wishes to model. Experimental evidences show as well that not individual but assemblages of grains detach during sand/solids production [3].

The presentation of results starts with the depiction of the plastic zone in the analyzed model after the excavation stage obtained both with Cosserat continua and with standard continua. As seen in Fig. 2, the differences are striking for different values of characteristic length and even more prominent when compared with the results obtained in a standard continuum. After the excavation stage, the plastic zone in the standard continua occupies an area of $0.02689 \mathrm{~m}^{2}$. By assuming a Cosserat continua and a characteristic length of $0.01 \mathrm{~m}$, this value increases to $0.03669 \mathrm{~m}^{2}$, and for a characteristic length of $0.02 \mathrm{~m}$, this increases to $0.04290 \mathrm{~m}^{2}$. Fig. 2 shows the deformed geometry with a scaling factor equal to 40 and not damaged zone is represented in blue. One is able to notice that in the case of standard continua, the borehole deformation occurs uniformly and radially, whereas in the Cosserat continua, a localization of deformation occurs around the borehole. This behavior has been found in laboratory tests, as shown in [41] for example. One can associate this mode of deformation with the rotations of the micro-structure, introduced by the anti-symmetric part of shear stress and also to the effect generated by the characteristic length, present in the second set of plastic flow and potential plastic functions $\left(f_{2}\right.$ and $g_{2}$; Eq. (17)), since both are triggered in this analysis. At this point, it should be observed that other authors have demonstrated a capability to numerically represent the behavior observed the laboratory without using specialized continua such as Cosserat continua. This is, for example, the case of analyses presented in [42]. These authors have analyzed similar cases as the ones presented here by using standard continua procedures. However, in order to achieve localization of deformation as shown here, Detournay [42] needed to add imperfections in the geometry and to impose conditions to the constitutive models adopted, such as the inclusion of softening or nonassociated plasticity in the case of hardening. Cosserat continua presents an alternative to standard continua in the sense that it does not need to introduce any of the above for localization. It is however true that Cosserat continua introduces additional parameters, which would have to be estimated or determined. Although not the main focus of the present work, another favorable aspect for the use of Cosserat continua in the analysis of elastoplastic problems or localization problems has to do with mesh dependency. According, for example to Sharbati and Naghdabadi [35] and Iordache and Willam [43], the introduction of Cosserat continua makes localization problems less dependent or even independent from the finite element meshes used. This is particularly important in the case of sand/solids production problems.

\section{a}

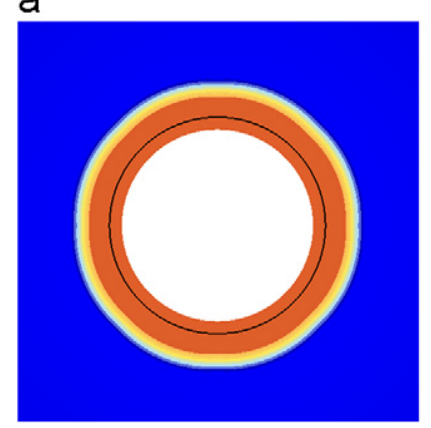

b

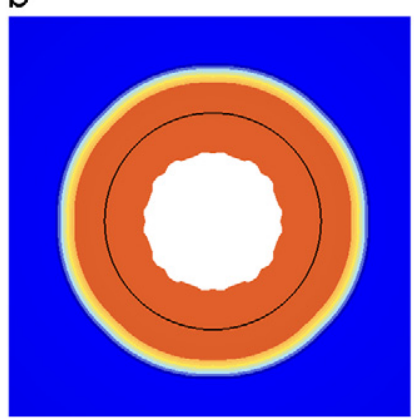

C

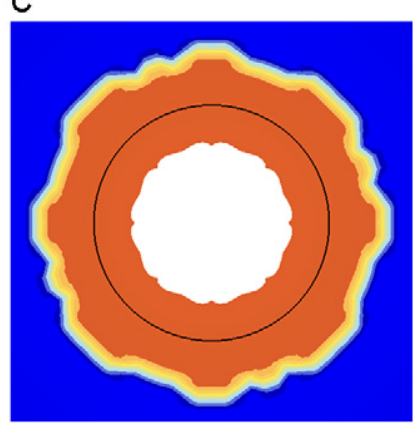

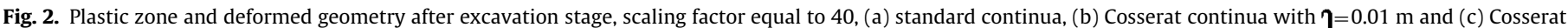
continuum with $\mathbf{\eta}=0.02 \mathrm{~m}$. (For interpretation of the references to color in this figure, the reader is referred to the web version of this article.) 
The characteristics shown in plastic zone after excavation phase had no interference from the sand/solids production process, showing only the importance of considering the Cosserat continua in this type of analysis.

Fig. 3 shows results corresponding to normalized deviatoric plastic strains after the excavation phase, together with the medium deformed geometry using a scale factor of 40 . These results show significant differences when considering Cosserat continua as compared to standard continua. In the former, as the characteristic length increases, the effect of localization of plastic deformations is accentuated.

Fig. 4 shows the results for the porosity for a time of $41 \mathrm{~h}$. Observe that in standard continua, the porosity presents a more homogeneous pattern than the ones obtained for Cosserat continua. One notes as well, in the case of Cosserat continua, more localized zones of porosity changes, their extension being larger with higher characteristic lengths. In Cosserat continua, porosity of the order of $87 \%$ are encountered, whereas in standard continua, these values are around $72 \%$.

The porosity field obtained after five days after excavation stage shown in Fig. 5, also shows different patterns. The results for the standard continua behave uniformly and radially. For the Cosserat continua, the porosity shows a less uniform behavior for a larger characteristic length. According to these results, the model studied tends to stabilize in this configuration, and can be considered that the region with a porosity of $98 \%$ was removed from the model.

Considering the zone that produced sand/solids and that after five days was completely removed from the model, one could expect that the pore pressures on the wall of the borehole also show and exhibit a consistent behavior with these results. Fig. 6 shows a plot of pore pressures extending radially away. One is able to notice that the radial variation in pore pressures depends on the type of continua being considered. a

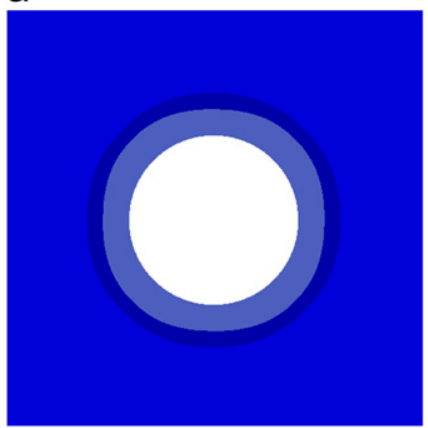

b

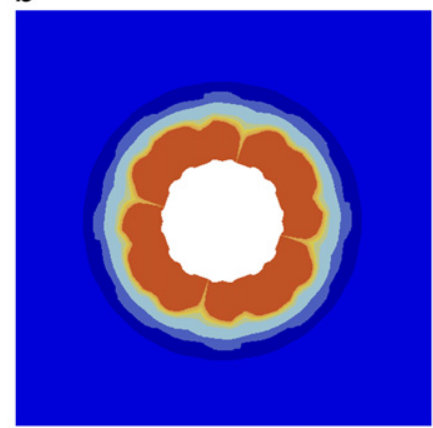

C

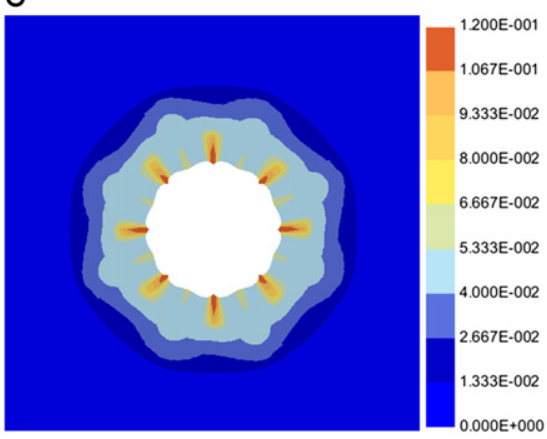

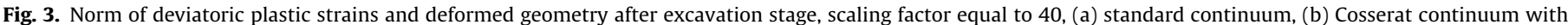
$\boldsymbol{\eta}=0.01 \mathrm{~m}$ and (c) Cosserat continuum with $\boldsymbol{\eta}=0.02 \mathrm{~m}$.

a

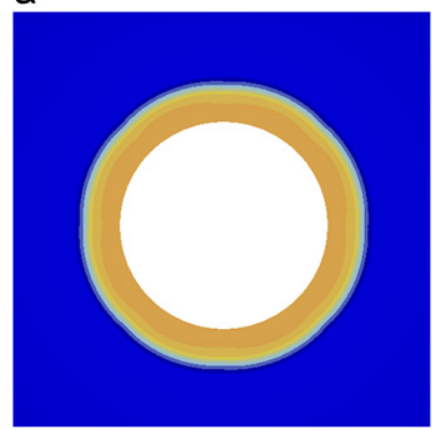

b

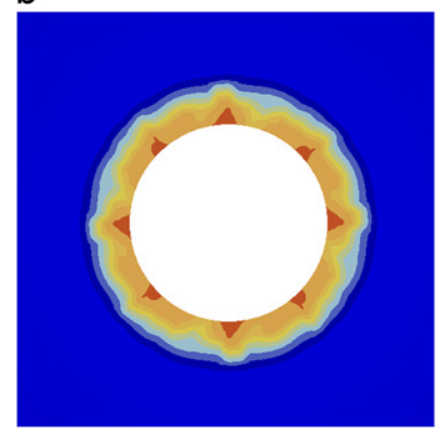

C

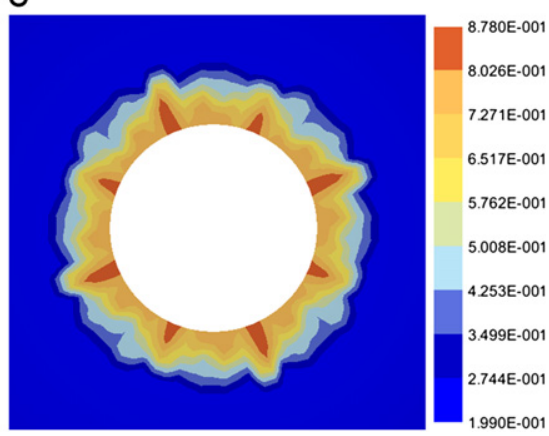

Fig. 4. Porosity $41 \mathrm{~h}$ after excavation stage, (a) standard continua, (b) Cosserat continuum with $\mathfrak{\eta}=0.01 \mathrm{~m}$ and (c) Cosserat continuum with $\mathbf{\eta}=0.02 \mathrm{~m}$.

a

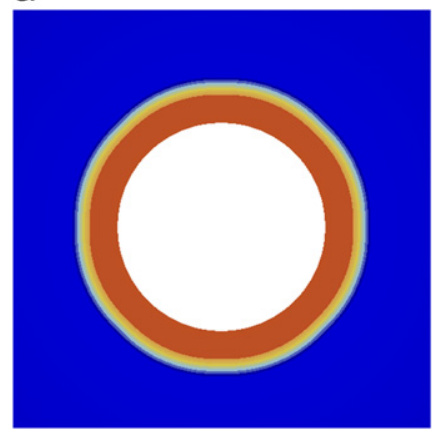

b

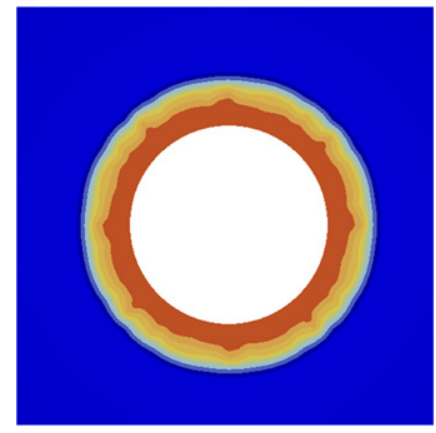

C

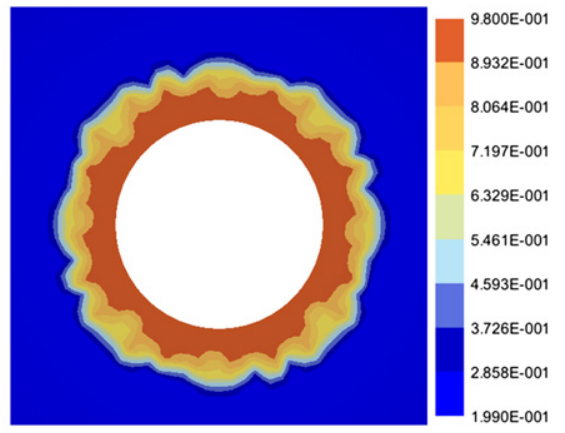

Fig. 5. Porosity 5 days after excavation stage, (a) standard continua, (b) Cosserat continuum with $\mathbf{\eta}=0.01 \mathrm{~m}$ and (c) Cosserat continuum with $\mathfrak{\eta}=0.02 \mathrm{~m}$. 
Another quantity, which is important in the calculation of sand/solids production according to the production model used in this work, is fluid velocity. Fig. 7 presents the results of the normalized fluid velocity five days after excavation stage. Note the higher values of velocity were obtained when considering the Cosserat continua and a characteristic length of $0.02 \mathrm{~m}$. Perhaps, more significant than the values obtained, is the pattern exhibited by the velocities around the borehole. Velocities present

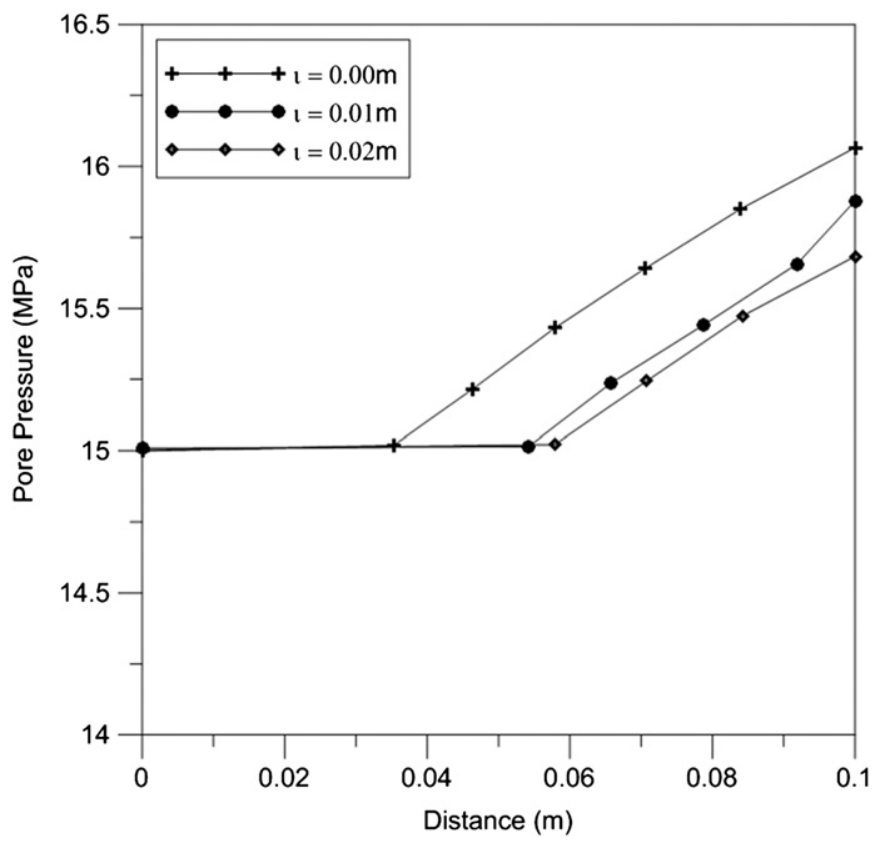

Fig. 6. Pore pressure vs. distance. a uniform and radial behavior in the case of standard continua. In the case of Cosserat continua, the pattern of velocities is rather more heterogeneous and localized. This is most certainly the consequence of strain localization in these models, which as a consequence produce localized changes in porosities and permeabilities.

Fig. 8 shows the behavior of maximum principal effective stresses around the borehole $41 \mathrm{~h}$ after excavation stage. The general picture is the same as observed with deformations shown previously. In the case of Cosserat continua, regions of stress concentration appear in a spiral form.

Fig. 9 presents the time evolution of sand/solids production volume being produced. Production volumes appear to be larger for Cosserat continua in comparison to standard continua. Production rates also appear to be slightly larger for the larger characteristic lengths Cosserat continua.

Figs. 3c and 4c present, respectively, localization patterns and porosities for the analyzed case. One is able to notice that the localization zones are located in eight diametrically opposed radial regions. The literature presents $[44,45]$ reports of experimental work where patterns such as the ones presented here were found. In other experimental works however, the localization patterns are restricted to two diametrically opposed radial regions as shown in Fig. 10 of [45].

This localization mode has been referred to as the slit type mode. A study was carried out in order to verify the possibilities of modeling this mode with the Cosserat implementation of the present work. The objective is therefore one of reducing the eight localized regions to two in a natural form, without significant perturbations of the model geometry and material parameters. For that purpose, a characteristic length of $0.02 \mathrm{~m}$ was considered.

The characteristic lengths of two diametrically opposed finite elements were slightly decreased. This decrease had the effect of activating sooner at these points the second yield surface of a

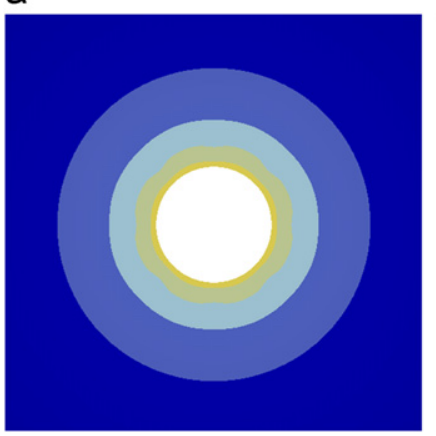

b

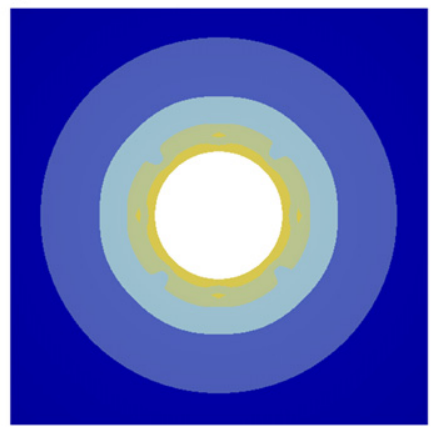

C

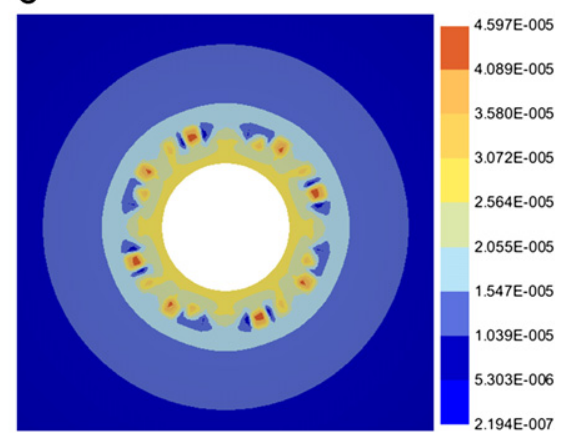

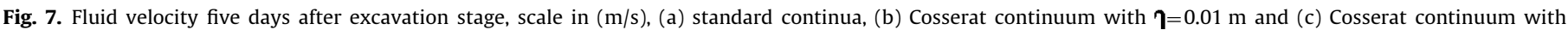
$\mathbf{\eta}=0.02 \mathrm{~m}$.

a

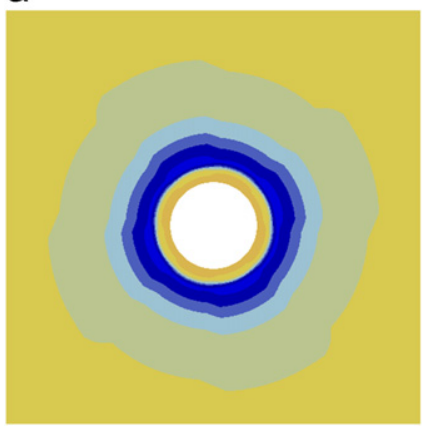

b

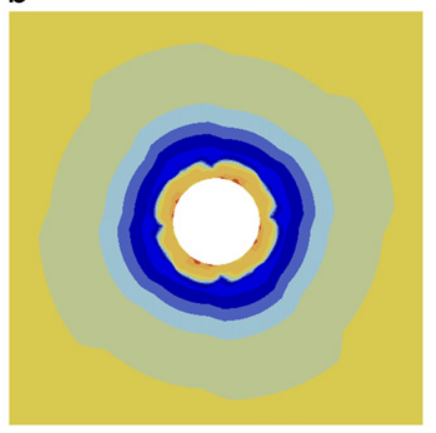

C

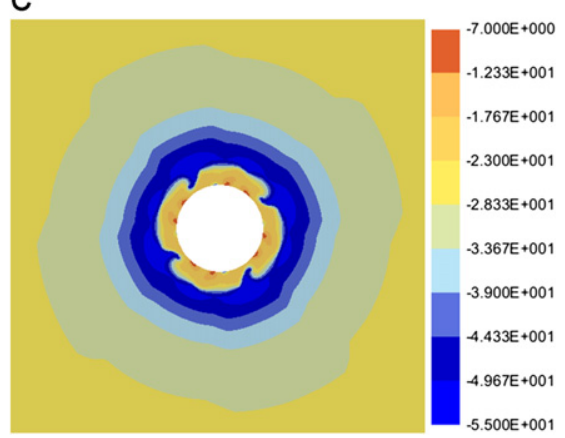

Fig. 8. Maximum principal effective stresses $41 \mathrm{~h}$ after excavation stage, scale in (MPa) (a) standard continuum, (b) Cosserat continuum $\mathfrak{\eta}=0.01 \mathrm{~m}$ and (c) $\mathfrak{\eta}=0.02 \mathrm{~m}$. 
Bogdanova-Bontcheva \& Lippmann's model presented in Eq. (17), the one related to couple stresses as mentioned above. However, the evolution of plastic zones and porosities continue to occur around the borehole surface as in the previously analyzed cases. In order to establish a preference zone for the evolution of porosities, a critical velocity $\|\mathbf{v}\|_{\text {critic }}=2.00 E-5 \mathrm{~m} / \mathrm{s}$ was established (instead of $\|\mathbf{v}\|_{\text {critic }}=0$ as in the previously analyzed cases). The impact of setting this threshold was to concentrate sand/ solids production in the zone around the points where failure by

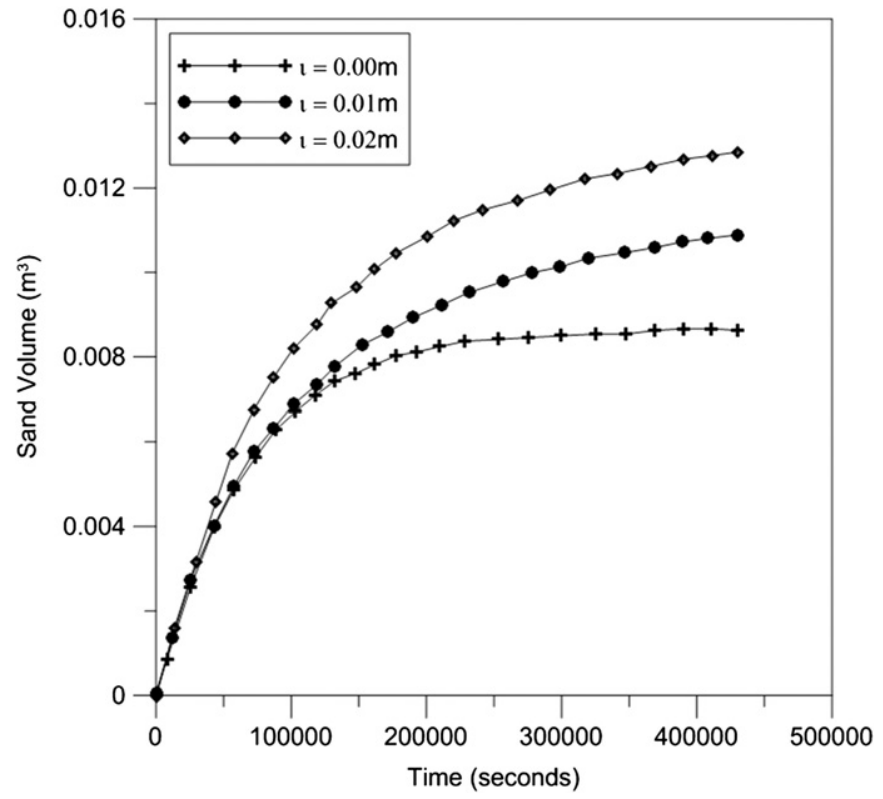

Fig. 9. Sand volume vs. time.
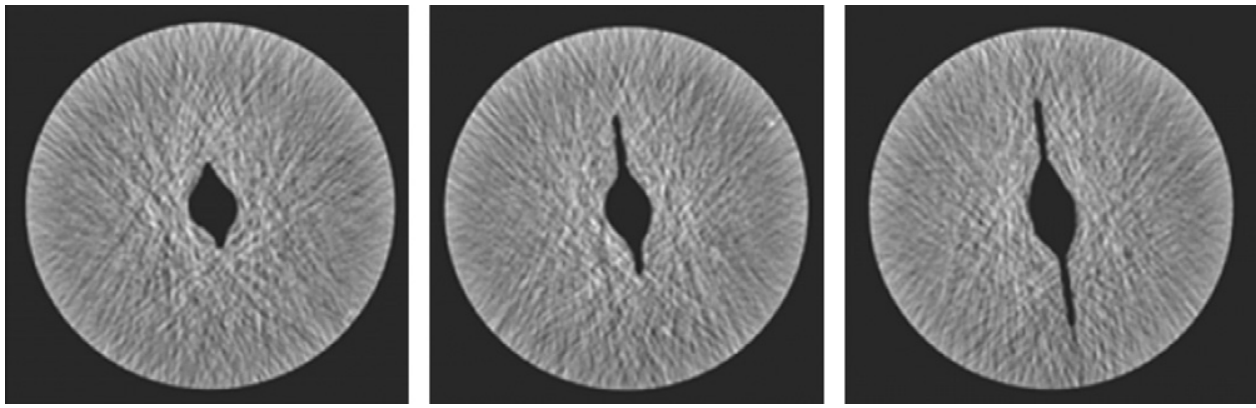

Fig. 10. States of development of slit type mode, from [40].

a

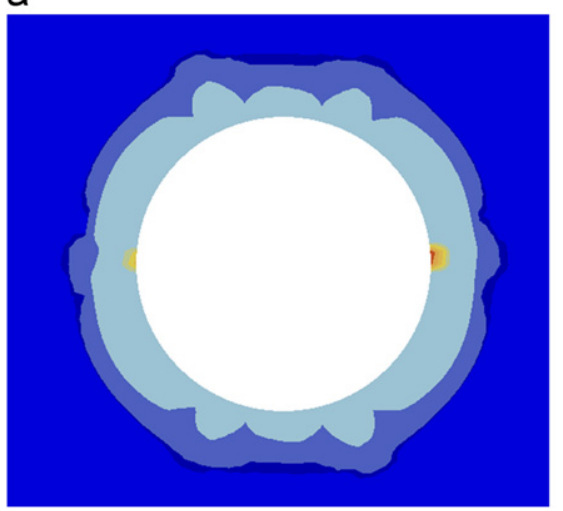

couple stresses started. Results for the norm of deviatoric plastic strains (defined in Section 2) and porosity $41 \mathrm{~h}$ after excavation stage are presented in Fig. 11. One is able to notice the localization of such quantities in a narrow zone characterizing what is called a slit type mechanism as shown in Fig. 10.

Fig. 12 presents sand/solids volume being produced for the analyzed case. One notes that the rate of sand/solids production is approximately constant up to a limit when the rock formation stops producing.

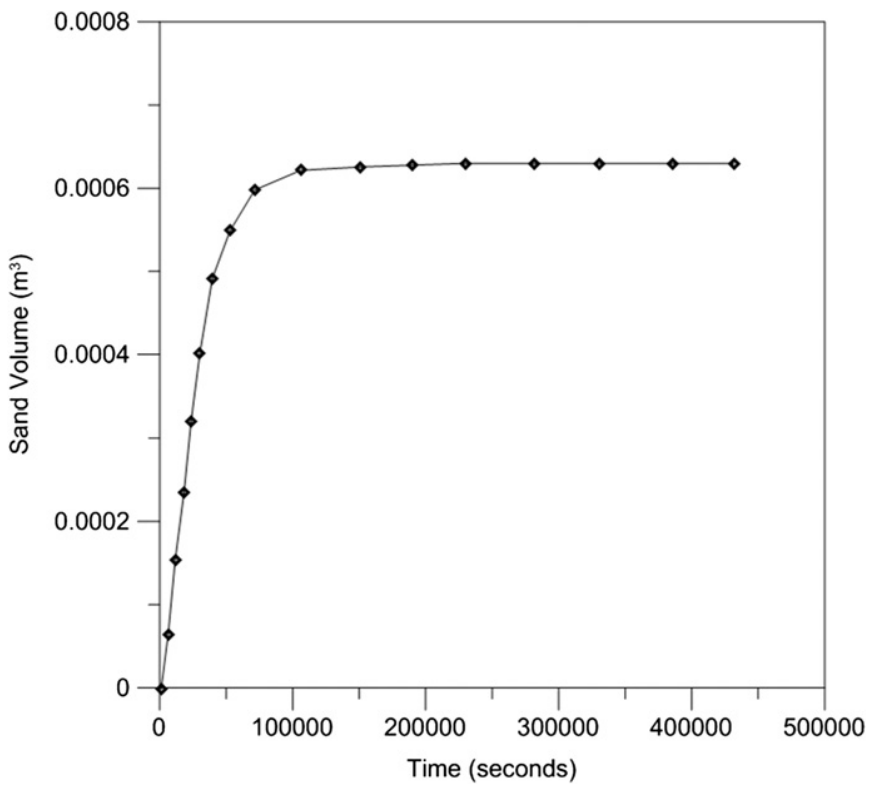

Fig. 12. Sand volume vs. time. b

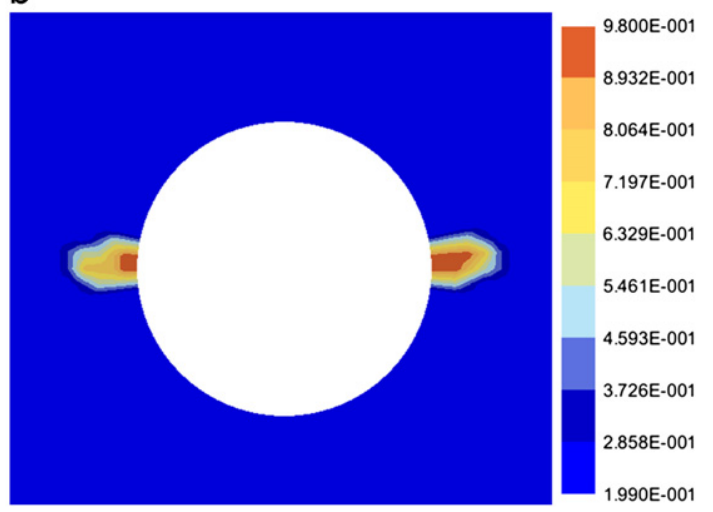

Fig. 11. (a) Norm of deviatoric plastic strains $41 \mathrm{~h}$ after excavation stage. (b) Porosity $41 \mathrm{~h}$ after excavation stage. 
a

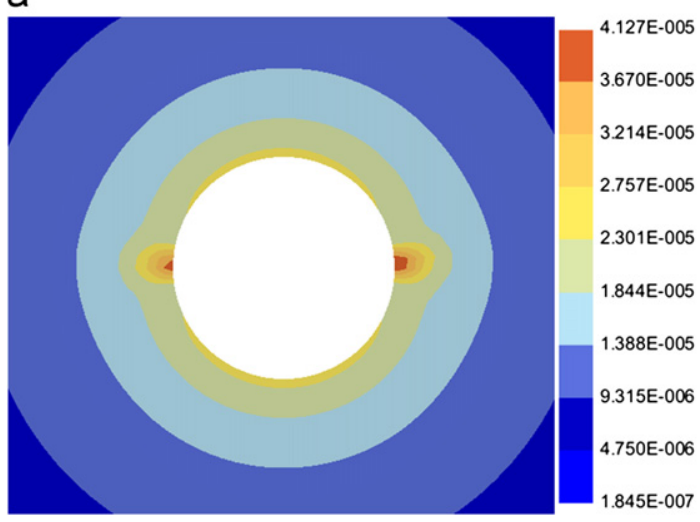

b

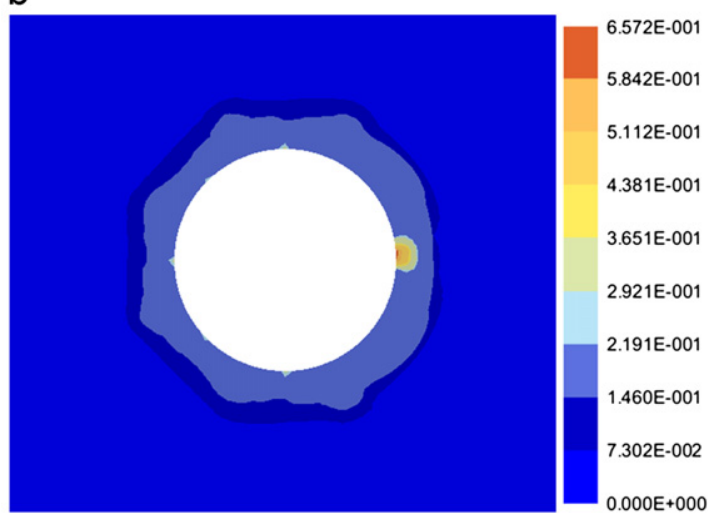

Fig. 13. (a) Normalized fluid velocity $1.5 \mathrm{~h}$ after excavation stage, scale in (m/s). (b) Norm of deviatoric plastic strains $1.5 \mathrm{~h}$ after excavation stage.

a

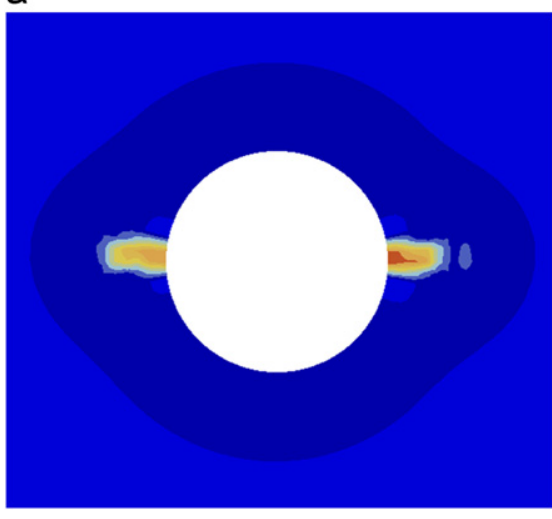

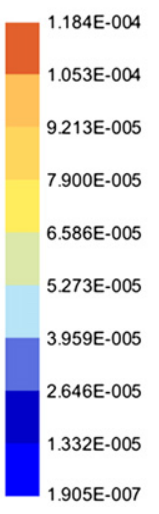

b

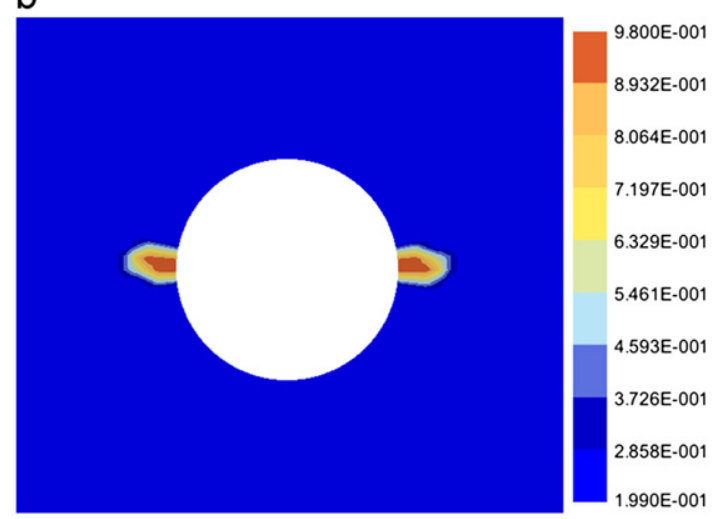

Fig. 14. (a) Normalized fluid velocity 5 days after excavation stage, scale in (m/s). (b) Porosity 5 days after excavation stage.

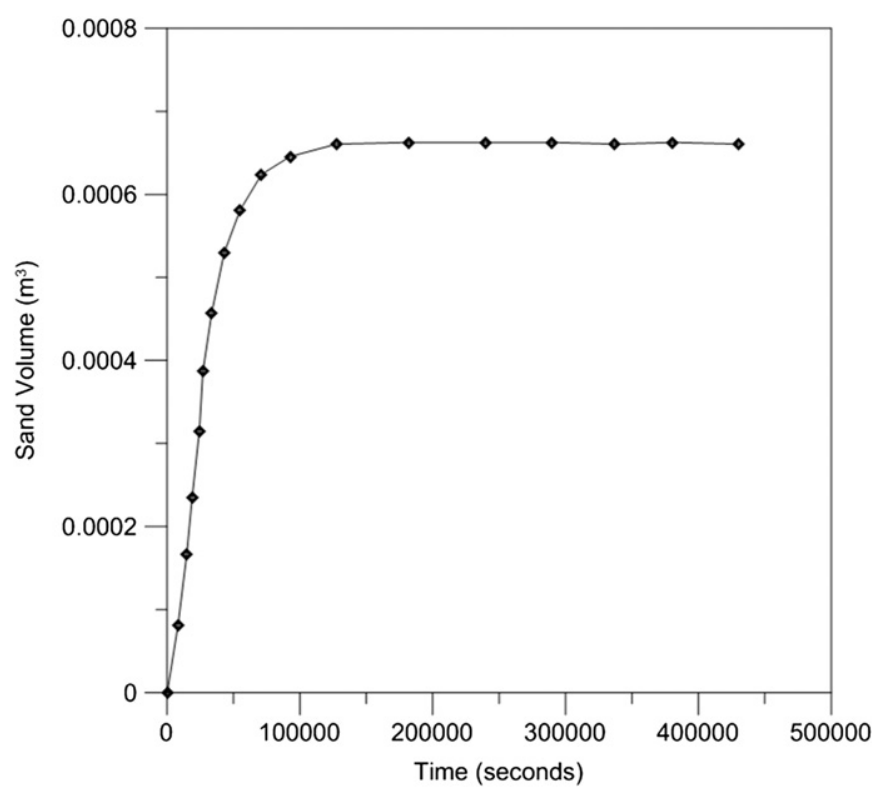

Fig. 15. Sand volume vs. time.

A further exercise was made in order to understand the formation of the slit mechanism. This time, instead of decreasing the characteristic length of two diametrically opposed elements, this was done only at one element around the borehole. The obtained results are shown in Figs. 13-15. What happened in this case was that deformations started localizing at this point as shown in Fig. 13, but soon enough and naturally started to localize at the diametrically opposed element as well. It appears therefore that, in order for this mechanism to start, one needs localization at one point around the borehole. The heterogeneous nature of real rocks could provide this condition. The sand/solids production volumes as shown in Fig. 15 are basically the same as in the previous case shown in Fig. 12. It is important to emphasize the relevance of this result. In the rock mechanics literature, it has been proposed that such mechanisms could be attributed to the formation of compaction bands, perpendicular to the in-situ maximum principal stresses. This hypotheses, however, does not apply to the situations analyzed in the present paper, that is, under a hydrostatic state of stresses. The results shown in Figs. 13 and 14 suggest that the appearance of plastic zones associated to micro-rotations could indeed be the real cause of these mechanisms. To the authors' knowledge, this suggestion for origin of slit type mechanisms had not yet appeared in the literature.

\section{Conclusions}

The development and application of finite element method for simulating the process of sand/solids production in boreholes considering the fluid-mechanic coupling and the use of an elastoplastic Cosserat continuum was presented. In order to model elasto-plastic behavior, the Bognanova-Bontcheva \& Lippmann's constitutive model was used. In order to evaluate the level of importance of Cosserat continua in sand/solids production 
prediction studies, different characteristic lengths of the rocks were considered. A hypothetical borehole was used as an example in order to illustrate the implemented procedures. The initial state of stress in the example was isotropic, which allowed qualitative comparisons with experimental work available for these conditions. As shown by other authors, the use of Cosserat continua provides a framework in order to model strain localization problems. Furthermore and also shown in the literature, sand/solids prediction can be extremely dependent on the failure/ deformational modes happening in the vicinity of boreholes. Obtained results in the present paper showed that this is indeed the case. The paper also presented a successful attempt to model in a natural way slit type failure around the borehole using the implemented models and the consequent prediction of sand/ solids being produced. Work to compare the numerical results using the proposed models with experimental results will be crucial for its validation. The paper showed that the consideration of Cosserat continua can indeed provide an appropriate framework for the numerical prediction of sand/solids prediction.

\section{Acknowledgements}

The authors thank Petrobras (Petróleo Brasileiro S/A) for funding the research reported in the present paper and Erling Fjaer for his critical comments and careful review of the paper.

\section{References}

[1] Dusseault MB, Santarelli FJ A conceptual model for massive solids production in poorly-consolidated sandstones. Rock at great depth, vol. 2; 1989. p. 789-97.

[2] Fjaer E, Holt RM, Horsrud P, Raaen AM, Risnes R. Petroleum related rock mechanics. Amsterdam: Elsevier; 1992.

[3] Bianco LC. Phenomena of sand production in non-consolidated sandstones. $\mathrm{PhD}$ thesis. Penn State University, University Park, Penn; 1999.

[4] Toma P, Harris B, Korpany G, Bohun D, George A. Experimental investigations for reducing the risk of sand inflow in slotted horizontal wells. In: Proceedings of the ASME energy source technical conference and exhibition, New Orleans; 1994. p. 1-10

[5] Geilikman MB, Dusseault MB. Dynamics of wormholes and enhancement of fluid production. In: Proceedings of the 48th annual technical meeting of the petroleum society, Calgary; 1997. p. 8-11.

[6] Unander TE, Papamichos E, Trovoll J, Skjaerstein A. Flow geometry effects on sand production from an oil producing perforation cavity. Int J Rock Mech Min Sci 1997:34:293E1-15.

[7] Morita N Field and laboratory verification of sand production models. In: Proceedings of the technical conference of the society of petroleum engineering, paper SPE 27341; 1994.

[8] Tronvoll J, Skjaerstein A, Papamichos E. Sand production: mechanical failure or hydrodynamic erosion? Int J Rock Mech Min Sci 1997;34:291E1-17.

[9] Papamichos E, Tronvoll J, Skjærstein A, Unander TE. Hole stability of Red Wildmoor sandstone under anisotropic stresses and sand production criterion. J Petrol Sci Eng 2010;72:78-92.

[10] Stavropoulou M, Papanastasiou P, Vardoulakis I. Int J Numer Anal Meth Geomech 1998:22:749-69.

[11] Papamichos E, Vardoulakis I. Comput Geotech 2005;32:47-58.

[12] Wang J, Wan RG, Settari A, Walters D. Prediction of volumetric sand production and wellbore stability analysis of a well at different completion schemes. In: Proceedings of the 40th US rock mechanics symposium, Anchorage; 2005.

[13] Liu Y, Wan RG. Computing sand production under foamy oil flow in porous media via least-squares finite elements. Finite Elem Anal Des 2006;42: 592-601.

[14] Detournay C, Tan C, Wu B. Modeling the mechanism and rate of sand production using FLAC. In: Proceedings of the 4th international FLAC symposium on numerical modelling in geomechanics, Minneapolis; 2006.
[15] Detournay C. Numerical modeling of the slit model of cavity evolution associated with sand production. SPE J 2008;14(4):797-804.

[16] Papamichos E. Erosion and multiphase flow in porous media: Application to sand production. Eur J Environ Civ Eng 2010;14:1129-54.

[17] Papamichos E, Vardoulakis I, Tronvoll J, Skjærstein A. Volumetric sand production model and experiment. Int J Numer Anal Meth Geomech 2001;25:789-808.

[18] Papamichos E, Malmanger EMA. sand erosion model for volumetric sand predictions in a North Sea reservoir. SPE Reser Eval Eng 2001;4:44-50.

[19] O'Connor RM, Torczynski JR, Preece DS, Klosek JT, Williams JR. Discrete element modeling of sand production. Int J Rock Mech Min Sci 1997;34: 231E1-15.

[20] Cook BK, Lee MY, DiGiovanni AA, Bronowski DR, Perkins ED, Williams JR. Discrete element modeling applied to laboratory simulation of near-wellbore mechanics. Int J Geomech 2004;4:9-27.

[21] Li L, Papamichos E, Cerasi P. A study of mechanisms of sand production using DEM with fluid flow. In: Cotthem AV, editor. Proceedings of the Eurock 06, Liege; 2006, pp. 241-247.

22] Cosserat E, Cosserat F. Theorie des Corps Deformables. Paris: A Hermann et Fils; 1909.

[23] Mindlin RD. Stress functions for a Cosserat continuum. Int J Solid Struct 1965;1:265-71.

24] Bogdanova-Bontcheva N, Lippmann H. Rotationssymmetrisches ebenes Flie \ssen eines granularen Modellmaterials. Acta Mech 1975;21:93-113.

[25] Kanatani K. A micro-polar continuum theory for the flow of granular materials. Int J Eng Sci 1979;17:419-32.

[26] Mühlhaus HB, Vardoulakis I. The thickness of shear bands in granular materials. Géotechnique 1987;37:271-83.

[27] Chang CS, Ma L. A micromechanical-based micropolar theory for deformation of granular solids. Int J Solid Struct 1991;28:67-86.

[28] Bardet JP, Vardoulakis I. The asymmetry of stress in granular media. Int J Solid Struct 2001;38:353-67.

[29] Muller AL, Vargas EA, Vaz LE, Gonçalves CJ. Borehole stability analysis considering spatial variability and poroelastoplasticity. Int J Rock Mech Min Sci 2009;46:90-6.

[30] Muller AL, Vargas EA, Vaz LE, Gonçalves CJ. Three-dimensional analysis of boreholes considering spatial variability of properties and poroelastoplasticity. J Petrol Sci Eng 2009;68:268-76.

[31] Lewis RW, Schrefler BA. The finite element method in the deformation and consolidation of porous media. London: Wiley; 1998.

[32] Providas E, Kattis MA. Finite element method in plane Cosserat elasticity. Comp Struct 2002;80:2059-69.

[33] Hongwu Z, Hui W, Guozhen L. Quadrilateral isoparametric finite elements for plane elastic Cosserat bodies. Acta Mech Sinica 2005;21:388-94.

[34] Vardoulakis I, Stavropoulou M, Papanastasiou P. Hydromechanical aspects of the sand production problem. Transp Porous Media 1996;22:225-44.

[35] Sharbati E, Naghdabadi R. Computational aspects of the Cosserat finite element analysis of localization phenomena. Comp Mater Sci 2006;38: 303-15.

[36] Hughes TJR. Unconditionally stable algorithms for nonlinear heat conduction. Comp Meth Appl Mech Eng 1977;10:135-9.

[37] Unterreiner P. Contribution à létude et à la dodélisation numérique des sols cloués: application au calcul en deformation des ouvrages de soutènement. Phd these, École Nationale des Ponts et Chaussées, Paris; 1995.

[38] Lippmann H. Eine Cosserat-Theorie dês plastischen Flie \ssen. Acta Mech $1969 ; 8: 255-84$

[39] Bendsøe MP, Kikuchi N. Generating optimal topologies in structural design using homogenization method. Comp Meth Appl Mech Eng 1988;71: 197-224.

[40] Bendsøe MP. Optimal shape design as a material distribution problem. Struct Optim 1989;1:193-202.

[41] Van Den Hoek PJ. Prediction of different types of cavity failure using bifurcation theory. In: Proceedings of the 38th US rock mechanics symposium, Washington DC, Paper ARMA 01-0045; 7-10 July 2001.

42] Detournay C, Wu B, Tan C. Hydro-mechanical modeling of damage around borehole in laboratory experiments. In: Proceedings of the 6th North American rock mechanics symposium, Houston, paper ARMA 04-590; 5-9 June 2004.

[43] Iordache M, Willam K. Localized failure analysis in elastoplastic Cosserat continua. Comput Meth Appl Mech Eng 1998;151:559-86.

[44] Papamichos E. Sand production. Physical and experimental evidence Geomech. Energy Prod. 2006:803-16.

[45] Papamichos E, Stenebråten J, Cerasi P, Lavrov A, Vardoulakis I, Fuh G-F, et al Rock type and hole failure pattern effects on sand production. In: Proceedings of the 42nd US rock mechanics symposium, San Francisco, paper ARMA 08217; 29 June-2 July 2008 